\title{
Three-year-old child head-neck finite element modelling: simulation of the interaction with airbag in frontal and side impact
}

\author{
F. Meyer and Rémy Willinger \\ Mechanical Institute of Fluids and Solids, \\ Strasbourg University, \\ 2 rue Boussingault, Strasbourg 67000, France \\ E-mail: meyer@imfs.u-strasbg.fr \\ E-mail: remy.willinger@imfs.u-strasbg.fr
}

\begin{abstract}
This study proposes to assess the interaction between the three-year-old child headneck system and a typical airbag, a protective system frequently used in the automotive field. Two separated models (head and neck) developed at the Strasbourg University (UDS) were coupled in order to estimate the injury risk during this type of impact. The first model developed is a three-year-old child finite element neck model (FEM) based on a realistic geometry. This FEM was validated in four directions against an original method based on scaling method. The second FEM is a three-year-old child head FE model published by Roth et al (2008). This model proposed an injury criterion in terms of Von Mises stress in the brain for moderate neurological injuries. After a coupling of these two FE models two impacts a frontal and lateral impact configuration are simulated. These impacts consisted of an airbag deployment at different gaps in order to calculate and estimate child brain injury risks.
\end{abstract}

Keywords: FEM; finite element model; head-neck; three-year-old child; airbag-head interaction; frontal/side impact.

\section{Introduction}

The growing demand for greater mobility in Europe has made individual transportation an essential and even inevitable feature of modern living. Children are more and more often conveyed in cars or other modes of road transportation. With this increased travel, the risk for children of becoming involved in an accident as occupant has consequently increased. Based on accident data, it is obvious that in spite of the significant improvements in recent years in vehicle safety, the current number of deaths and casualties added to the social and economic costs are still unacceptable. Fatalities and injuries, especially to children, should be reduced by all the available ways: public regulation, prevention/education of road users, road infrastructure, compatibility between vehicles, active, passive and tertiary safety devices. 
As regards children, it is very difficult to obtain figures for fatalities or severely injured children in the 27 European countries, but if we consider the EU 15 countries, where the use of child restraint has been mandatory for a long time, approximately 600 children are killed in cars on the European roads and 80,000 are injured annually (data source: IRTAD, 2004). There has been a huge effort on human adult finite element (FE) modelling, but very few attempts as far as children are concerned.

For ethical reasons, there is a paucity of experimental data concerning the child's head and neck characterisation. As a consequence, there is a considerable difficulty for the validation of children FE models. For the neck validation one solution is to use the scaling method established by Irwin and Mertz (1997). This method permits to calculate a theoretical experimental corridor based on the adult experimental data, in terms of displacement and acceleration. The mechanical properties such as the mass density of the cervical vertebrae, the rigidity both for the intervertebral discs and the ligament are calculated with this scaling method.

One way to investigate child head injury criteria using numerical models is to simulate real world head trauma. Well-documented accidents can help us to understand child injuries in comparing numerical mechanical parameters with what really happened, distinguishing the biofidelic behaviour of a child numerical head and the ability to have an injury-predicting tool. Indeed, even if the biofidelic behaviour of child models cannot be checked, based on classical experimental vs. numerical validation process, investigations of child injury mechanisms can be performed by developing an injury-predicting tool, studying numerical simulation of a large number of real accidents, and correlating mechanical parameter outputs with observed injuries. In the present work, these previous published head and neck models are coupled to a simplified thorax in order to investigate child head-neck response under frontal and lateral airbag deployment as a function of initial distance between airbag and head.

\section{Materials and methods}

\subsection{Three-year-old child's neck FE model}

The neck model used in the present study has been previously published (Meyer et al., 2008) and will therefore be presented very briefly.

A three-year-old male child head and neck were scanned (Figure 1) in order to base this study on a realistic human geometry, and to integrate the detailed vertebrae anatomy. The surfaces were reconstructed, so that the cervical vertebrae could be completely meshed (Figure 2).

For the cervical vertebrae, shell elements offer the possibility of strictly respecting the anatomical surface. The upper and the lower ligamentary system were reproduced with springs elements and the intervertebral discs with bricks elements three layers). Finally, the model of the three-year-old child neck contains a total of 2,826 brick elements and 44,758 shell elements and 712 springs. 
Figure 1 Reconstruction of the cervical spine based on scanner section

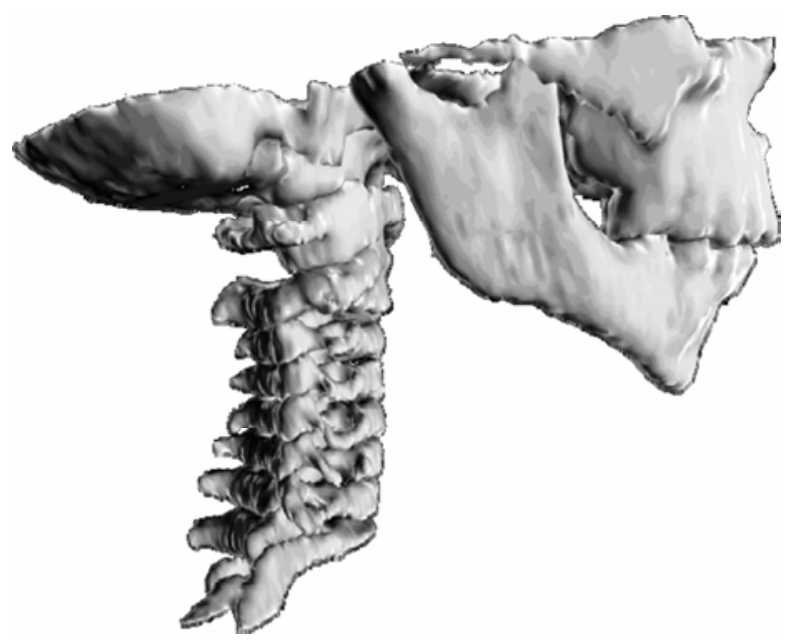

Figure 2 Surface meshing of the cervical column (C1-T1) with its ligamentary system

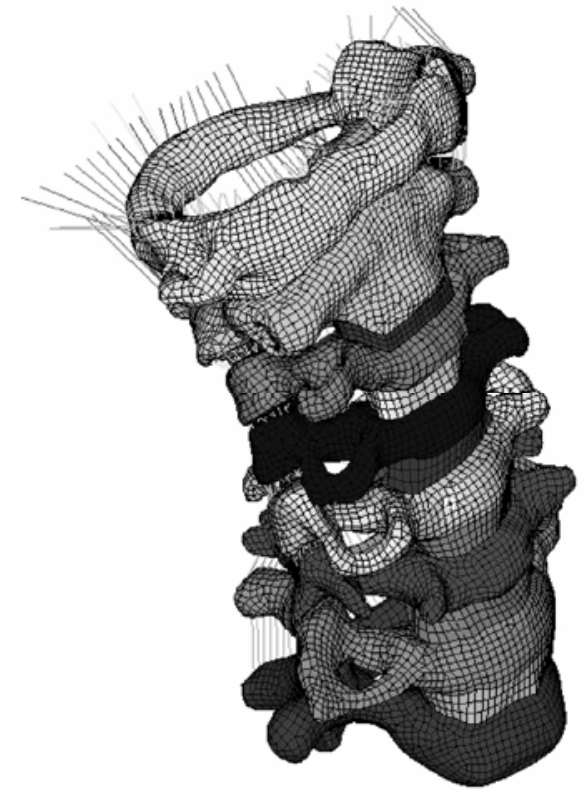

Source: See Meyer et al. (2008) for further details.

Finite element models (FEMs) of adult neck are typically validated against experimental data carried out by the NBDL (Meyer et al., 2004; Van der Horst, 2002) with frontal, oblique and lateral impacts (Ewing et al., 1968, 1977). Unfortunately, for ethical reasons, it is not possible to perform similar tests on children so no data exist in the literature for dynamic validation of a paediatric neck model. In the present study, inputs for the three-year-old-child model correspond to those used in the NBDL tests (frontal, lateral 
and oblique) but outputs, that is, head accelerations and displacement corridors, are scaled down in accordance with Irwin's method (Irwin and Mertz, 1997). An example of the frontal validation is illustrated in Figure 3, where the superimposition of experimental response corridors obtained with the scaling method, and numerical curves obtained with the FEM of the child neck is reported.

Figure 3 Results under frontal impact: $x$-axis (a), $z$-axis (b) linear head acceleration, $x$-axis (c), $z$-axis (d) head displacement and kinematics response of the whole head/neck system (e)
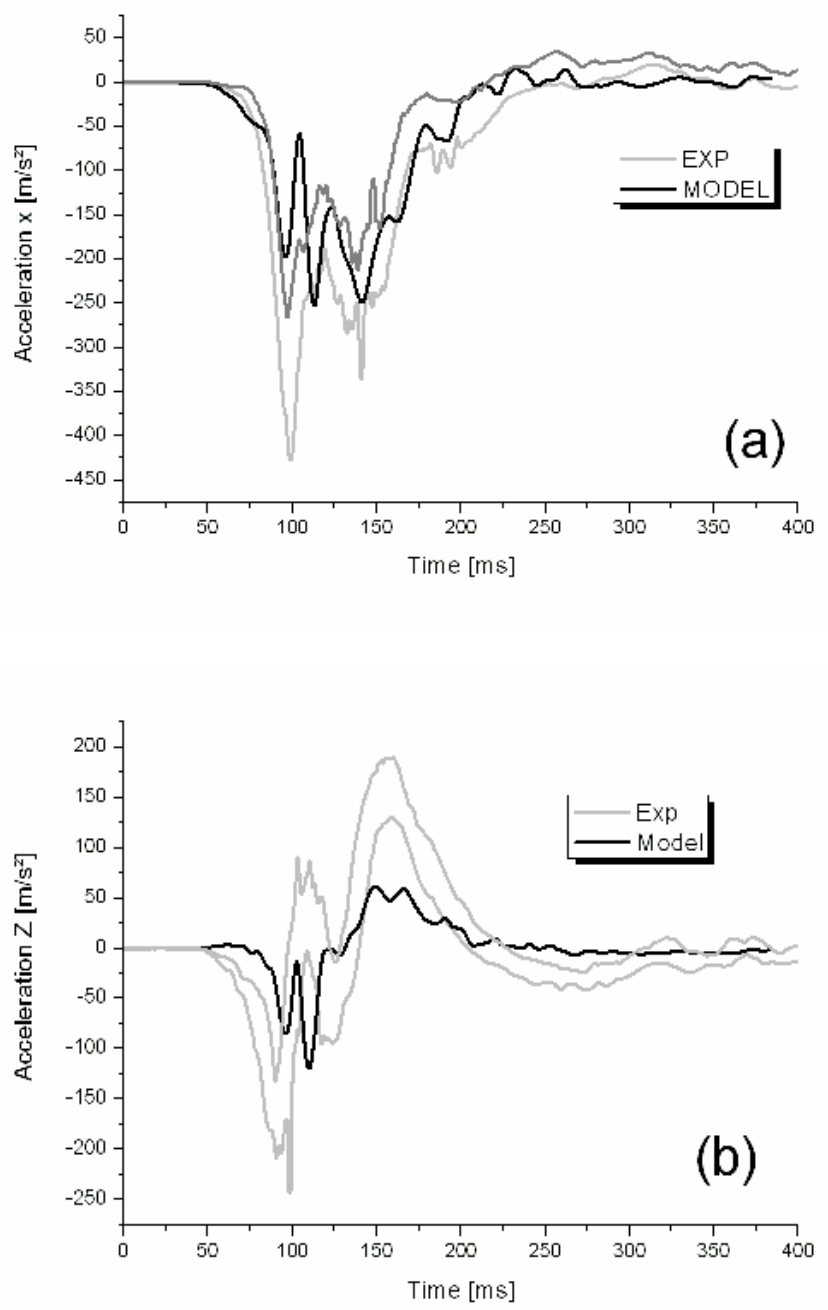
Figure 3 Results under frontal impact: $x$-axis (a), $z$-axis (b) linear head acceleration, $x$-axis (c), $z$-axis (d) head displacement and kinematics response of the whole head/neck system (e) (continued)
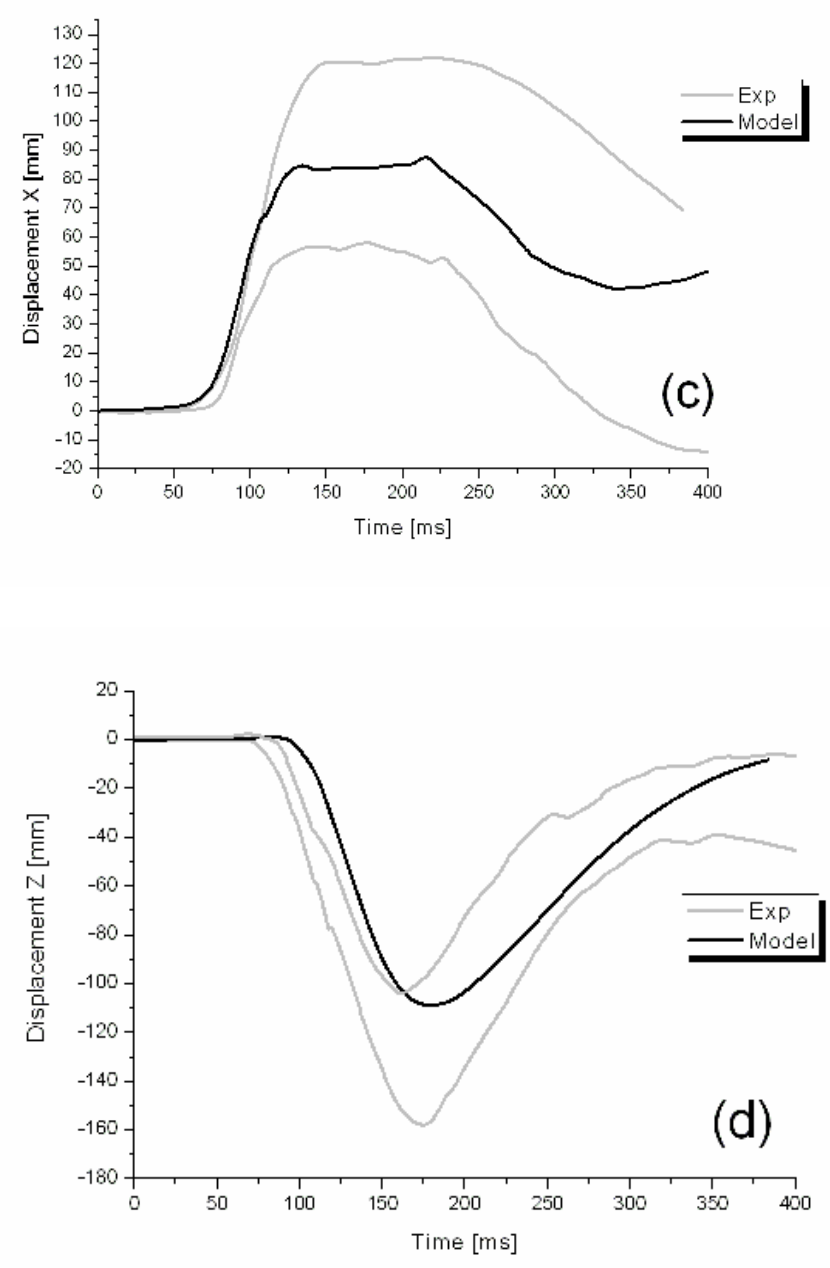

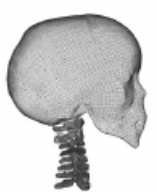

Time $=0 \mathrm{~ms}$

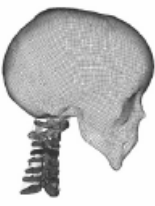

Time $=95 \mathrm{~ms}$

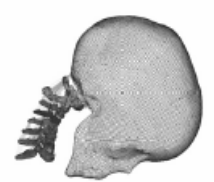

Time $=130 \mathrm{~ms}$

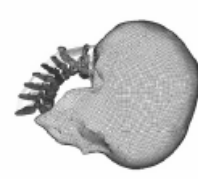

Time $=160 \mathrm{~ms}$

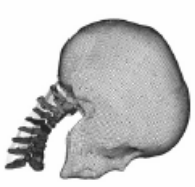

Time $=295 \mathrm{~ms}$

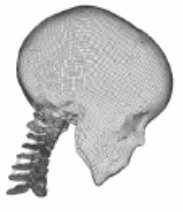

Time $=395 \mathrm{~ms}$ 


\subsection{Child's head model and injury criteria}

The head model that will be coupled to the neck was published by Roth et al. (2008). Hereafter, a short presentation is given.

As illustrated in Figure 4, the developed three-year-old head model takes into account the main anatomical features of a three-year-old child. It includes the scalp, the skull, the sutures (sagittal, coronal and lambdoid), the face, the cerebrospinal fluid (CSF), the falx, the tentorium and the brain. Finally, the whole model contains a total of 23,000 brick elements and 3,500 shell elements.

Figure 4 Meshing description of the detailed three-year-old child head FEM (a) cross section of the head FEM (b) membranes falx and tentorium

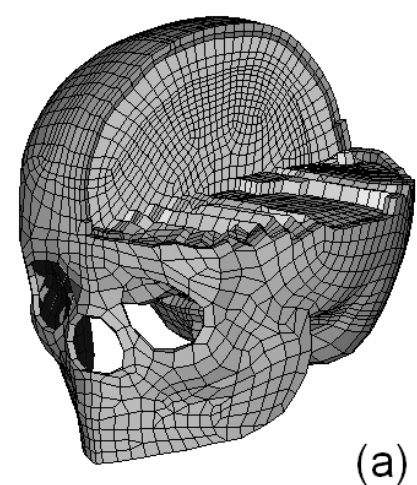

(a)

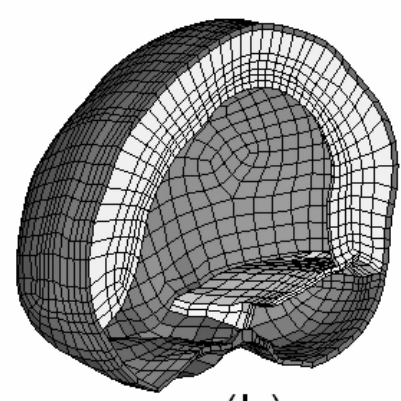

(b)

Source: See also Roth et al. (2008).

To investigate child injury criteria with the FEM, 25 real world accidents involving children aged from 2.5 to 3.5 years were collected. These accidents are free fall from different heights and are simulated with the 3 YOC head in order to extract the best mechanical parameter able to predict injury occurrence. From medical files, several data are available: gender, age, height of fall, type of impacted surface and type of injury. Injuries are classified into two categories: moderate neurological injuries (two in the AIS 
scale, unconsciousness limited to few hours after impact) and severe neurological injuries ( $>3$ in the AIS scale, with a $>24 \mathrm{hr}$ coma). Among these 25 cases, 15 accidents induced with no neurological injuries, 8 led to moderate neurological injuries and 2 to severe neurological injuries.

The determination of the head injury risk curves for specific injury mechanisms is based on a correlation study between the values of the proposed candidate criteria and the neurological lesions occurrences. Maximum values of mechanical parameters are used to build a histogram. The accident cases are finally sorted according to the injury classification, that is, moderate or absence of neurological injuries. When the injury predictor candidate is adequate, a distinction is visible between the low values of the uninjured cases and the high values of the computed for the injured cases. This threshold can accurately be calculated since it is the value leading to a 50\% risk an injury. For the statistical approach, the modified maximum likelihood method is chosen. It is a logistic regression method developed and described by Nakahira et al. (2000). The quality of the regression is thereby given by the negative estimator EB which should be as close to zero as possible. For each of these parameters: Von Mises stress, peak linear acceleration, maximum pressure, peak angular acceleration and HIC value, EB were calculated in order to identify the most relevant injury parameter (Figure 5).

As a result of numerical reconstructions of real world cases, shear distribution in term of stress appear to be an interesting predicting candidate for neurological lesions. These parameters had also been used for prediction of neurological injuries in adult head FEM by Deck and Willinger (2008). As a conclusion, a Von Mises brain shearing stress of $48 \mathrm{kPa}$ will be retained for neurological injuries (Figure 6).

Figure 5 EB regression parameters for several candidates for moderate neurological injury criterion

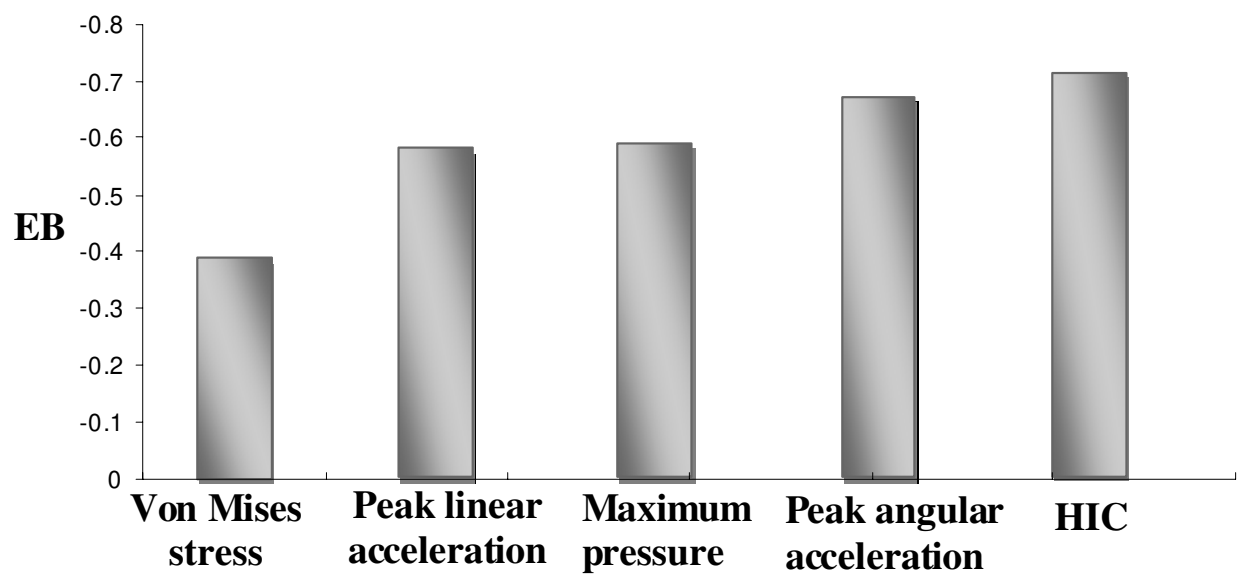


Figure 6 Histogram illustrating the correlation between the best mechanical parameter candidate (brain Von Mises stress) computed with three YOC FE model and corresponding injury risk curves

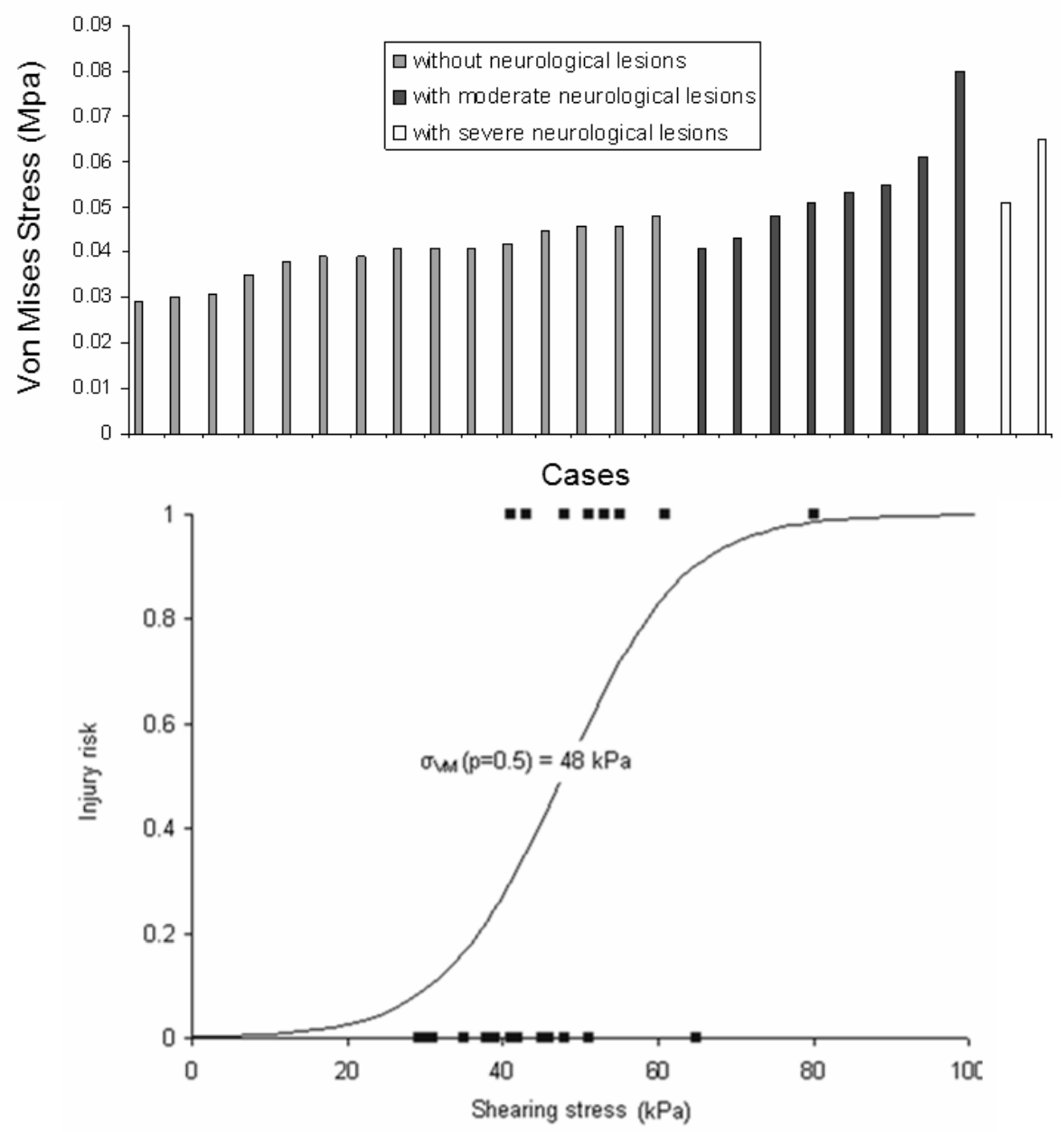

\subsection{Coupled three-year-child head-neck-thorax model under impact}

In the framework of the present study, the neck FE model was coupled to the head FE model. The connection between the head and the neck is made through the ligamentary system. The existing upper ligaments were connected to the head FEM and the contact between the atlas and the occiput was reproduced with a sliding interface. The objective of the coupling between the neck and the thorax was to take into account the mass and inertia effect of the thorax in case of an airbag impact. The geometry was taken from an adult thorax and scaled down in accordance with Irwin's method. As the thorax has only an inertial effect in the context of this study a very simplified thorax model is proposed. The whole three-year-old coupled model is illustrated in Figure 7. 
To provide realistic inertia, the thorax was meshed with bricks elements and the density was adjusted in order to have a mass of $6.61 \mathrm{~kg}$ (Irwin and Mertz, 1997) and an inertia of $I_{x x}=3.15 \times 10^{7} \mathrm{~g} \mathrm{~mm}^{2}, I_{y y}=2.73 \times 10^{7} \mathrm{~g} \mathrm{~mm}^{2}, I_{z z}=2.36 \times 10^{7} \mathrm{~g} \mathrm{~mm}^{2}$. Finally, the T1 vertebra was associated to the thorax as a key element for the coupling of this segment to the head-neck complex.

To simulate child airbag interaction during airbag deployment two impacts conditions are suggested, a frontal and a lateral one. For each case, the child is supposed to be seated statically without seat back, that is without any restrain of his thorax and with no initial velocity. For the frontal impact five distances between the chin and the airbag are proposed that is $6,8,10,12$ and $13.5 \mathrm{~cm}$. For the lateral impact five distances between ear and airbag are suggested as well, being $6,8,10,12$ and $13.5 \mathrm{~cm}$. For these 10 impacts the injury parameters at head will be computed in order to express the injury risk for each case. Figure 8 presents more details relatively to the initial conditions of this impact simulation for the frontal configuration, as the airbag centre of mass is set at $4.2 \mathrm{~cm}$ below the child head centre of mass.

Figure 7 Coupling of the three-year-old child head-neck FEM to a simplified thorax model

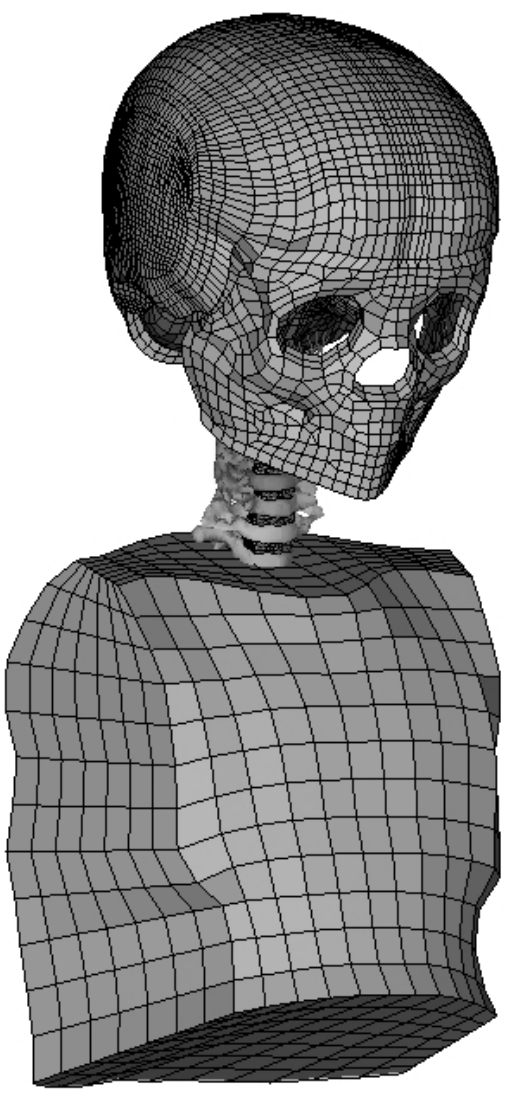


Figure 8 Child under airbag deployment under frontal configuration

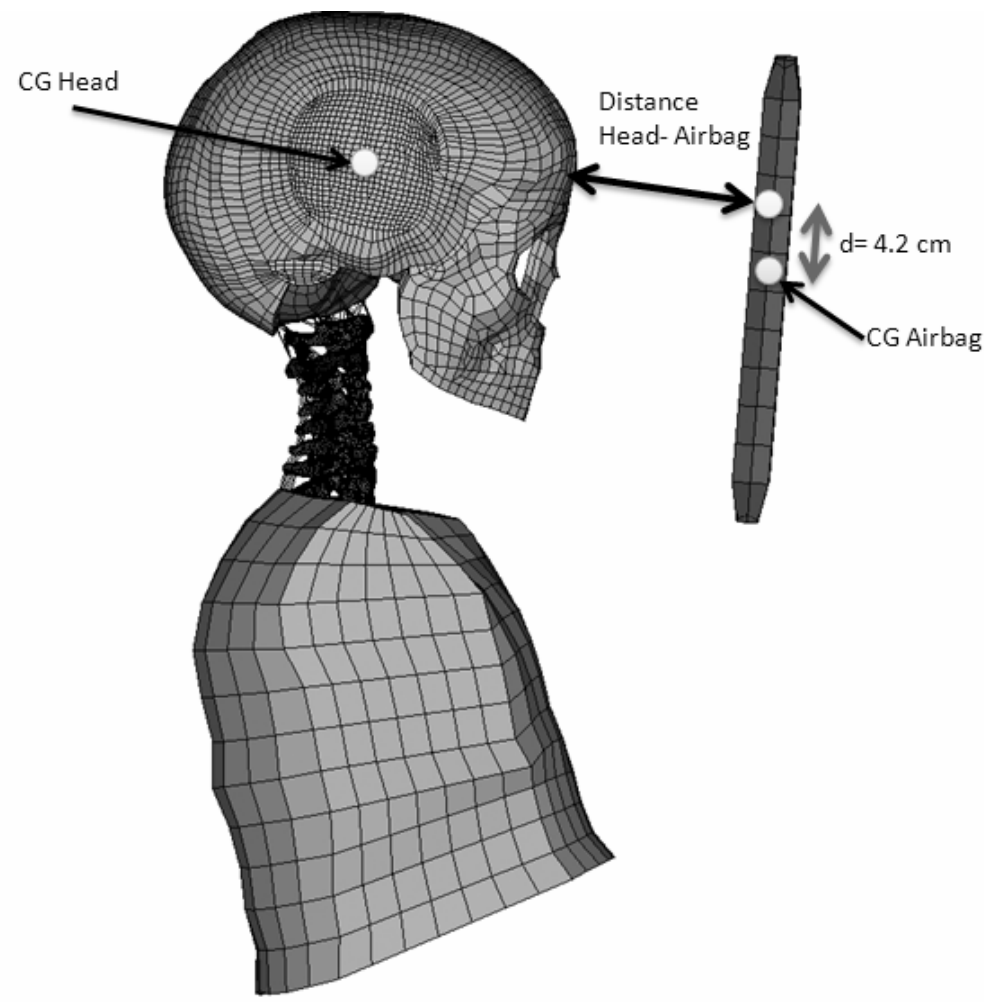

\section{Results}

\subsection{Frontal impact}

Figure 9 represents the maximum of forces calculated per head/airbag distances. It can be observed that there is no significant correlation between head/airbag distance and calculated maximum interaction force.

For the five airbag distances simulated the intracerebral Von Mises stress calculated (location and time evolution curves) are reported in Figure 10. The locations of these maxima are similar in the five cases (at the vertex area). Five bricks were considered to obtain a mean value of the time evolution of Von Mises stress at these maxima location. 
Figure 9 Maximum interaction force calculated for the five head-airbag distances $(6,8,10,12$, and $13.5 \mathrm{~cm}$ )

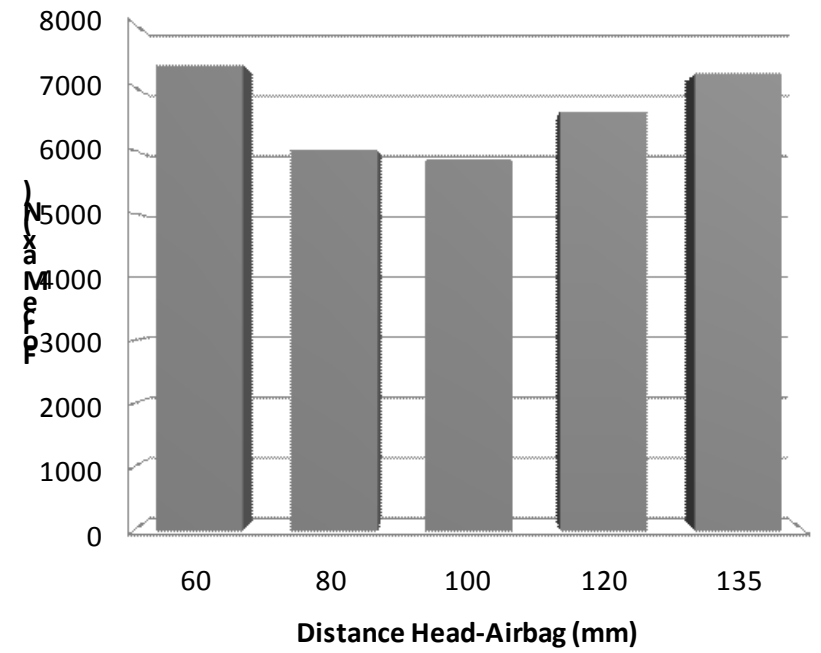

Figure 10 Illustration of the intracerebral Von Mises stress computed for the distance $100 \mathrm{~mm}$ (location of these maxima on left and time evolution on right) in frontal impact configuration
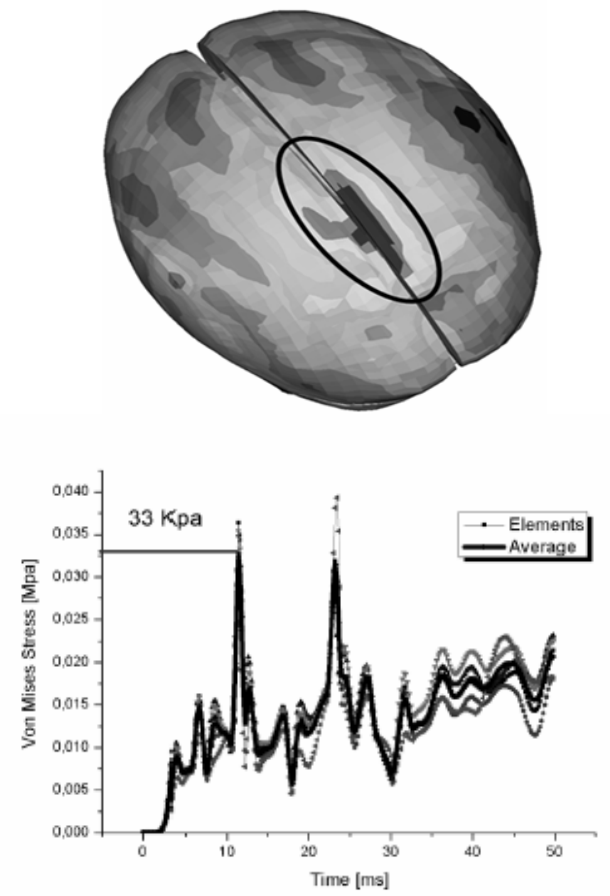
Figure 11 Maxima of intracerebral Von Mises stress computed within for the five head/airbag distances under frontal impact configuration

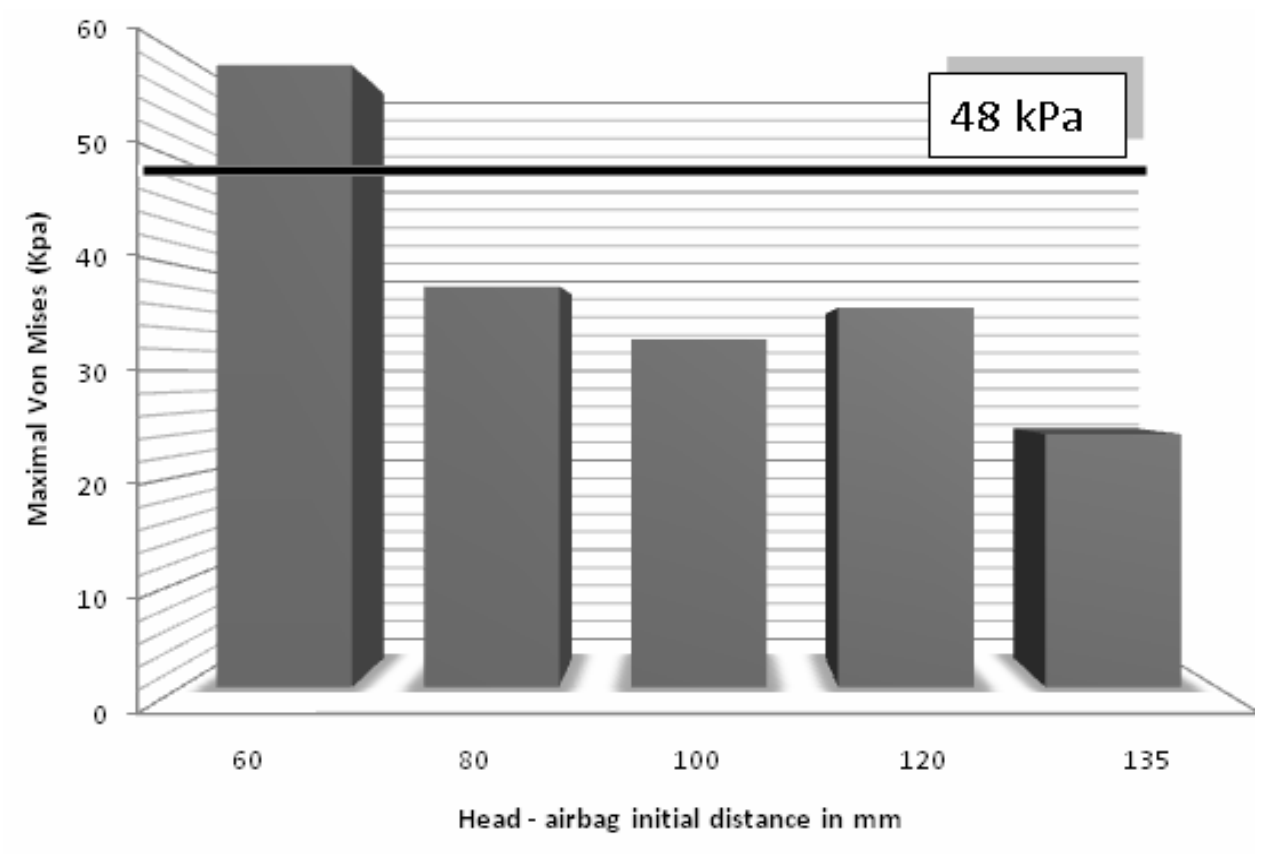

Maxima of Von Mises stress are obtained after the total airbag deployment (after $10 \mathrm{~ms}$ ) and all results are summarised in Figure 11.

For the head-airbag distance of about $60 \mathrm{~mm}$, an intracerebral Von Mises stress of $59 \mathrm{kPa}$, which is higher than the tolerance limits calculated for a $50 \%$ risk of moderate neurological injuries ( $48 \mathrm{kPa}$ ) is obtained. It is interesting to observe that the intracerebral Von Mises stresses are much more correlated with the head-airbag distance then the interaction force is.

\subsection{Lateral impact}

The conditions of the lateral airbag impact are similar to the frontal impact, that is, a free thorax boundary conditions. An overall view of the kinematics under this lateral airbag deployment $(d=100 \mathrm{~mm})$ is illustrated in Figure 12 .

As for frontal impact configuration no significant correlation between head/airbag distance and maximum interaction force calculated is observed as illustrated in Figure 13. 
Figure 12 Overall kinematics of the head FEM under lateral airbag impact

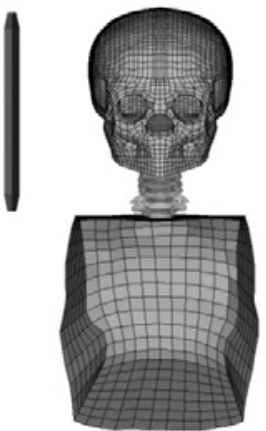

Time $=0 \mathrm{~ms}$

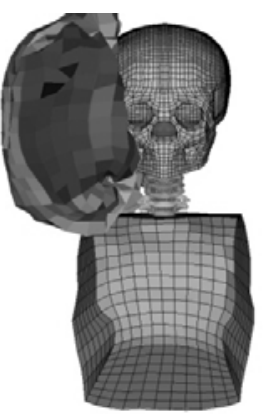

Time $=4 \mathrm{~ms}$

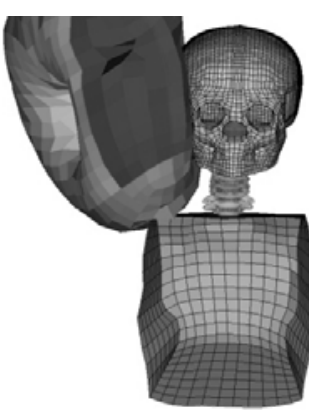

Time $=6 \mathrm{~ms}$

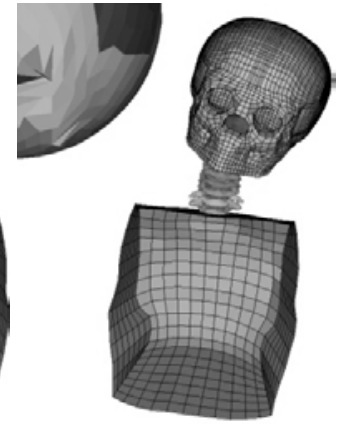

Time $=14 \mathrm{~ms}$

Figure 13 Maximal interaction force calculated for the five head-airbag distances $(60,80,100$, 120 and $135 \mathrm{~mm}$ )

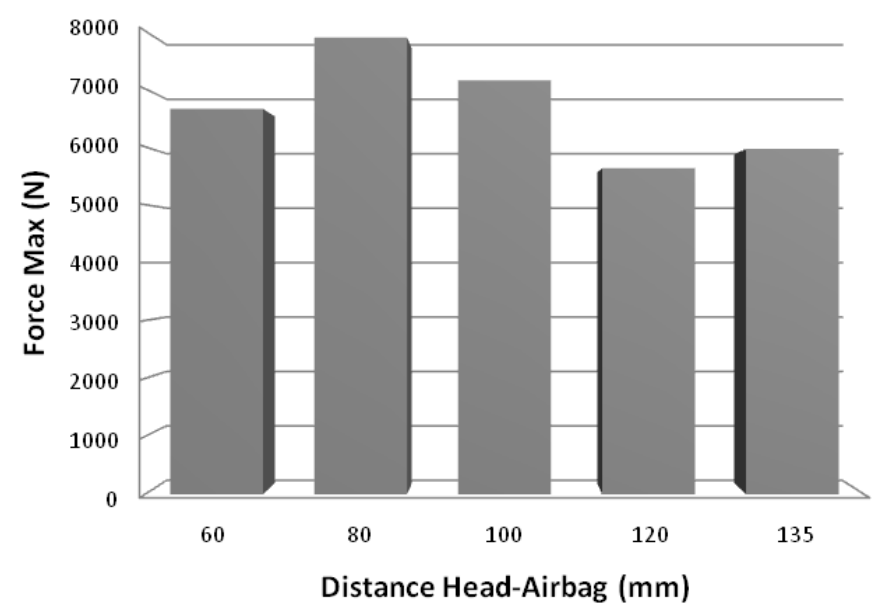

Figure 14 Maxima of intracerebral Von Mises stress computed within the brain for the five head/airbag under lateral impact configuration

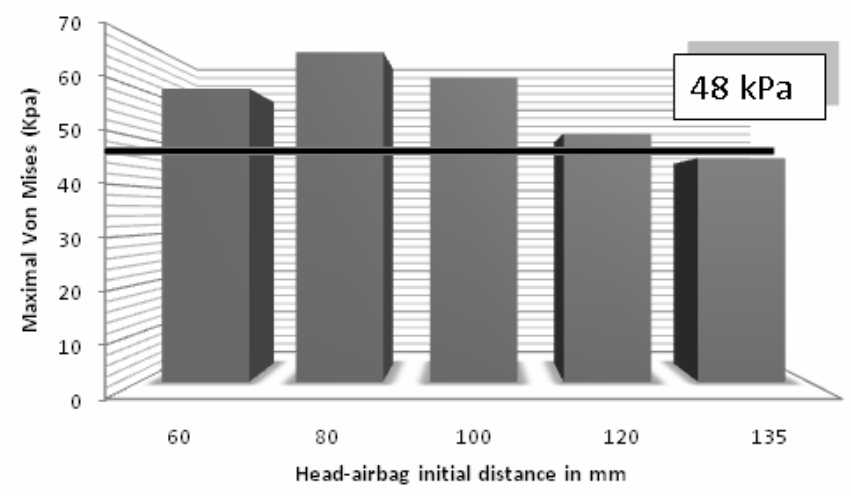


Figure 14 shows the maximum intracerebral Von Mises stress computed (location and time evolution curves) for the five airbag distances in lateral impact configuration. The locations of these maxima are similar in the five cases (at the opposite side to the impacted area). Except for the $135 \mathrm{~mm}$ distance, all simulated cases arrived to the same conclusion, that is, that there exists a risk of moderate neurological injury because the calculated intracerebral Von Mises stress exceeds the tolerance limit fixed to $48 \mathrm{kPa}$.

\section{Discussion}

After the development of a three-year-old child head-neck FE model and its use under airbag deployment, it is important to define the limitations of this study. A number of limitations exist at the biomechanical modelling level where clearly improvements are needed in the future, especially as far as neck injury criteria are concerned. The boundary conditions applied are not the same as in accident conditions as the simulations do not take into account the initial velocity of the whole body, the effect of the seatbelt and the initial position influence on the kinematics and the injury risk. The main originality of the proposed head-neck-thorax model is to consider a realistic and detailed geometry of the cervical spine and an FE head model with proposed tolerance limits for moderate neurological lesion. Therefore, it is a step towards numerical tools for the assessment of the child head and neck injury risk under airbag deployment.

\section{Conclusions}

The objective of this study was to assess the injury risk of the child head-neck system under airbag deployment for frontal and lateral configuration.

The study was based on a head and neck model of the three-year-old child developed in earlier studies, as well as first head injury criteria. Proposed then is the coupling of the head-neck system to a simplified thorax model in order to assess head injury risk under frontal and lateral airbag deployment. A parametric study on head-airbag distance was conducted with following main conclusions.

For the frontal airbag deployment, it was shown that there is a low correlation between initial distance and the interaction force. A head injury risk appears only if the initial distance between the airbag and the head is less than $80 \mathrm{~mm}$.

Concerning the lateral airbag deployment configuration, a similar conclusion can be made as for the frontal impact, that is, no correlation between the interaction force and the injury risk. However, the brain injury risk appears to be much higher than for the frontal impact. For all distances a brain injury risk of over $50 \%$ has been computed.

Even if a number of limitations exist in this child response simulation under impact, the first steps have been provided towards numerical tools designed for the assessment of child head-neck injury risk under deployment.

\section{Acknowledgements}

The authors wish to thank TRW for their support. 


\section{References}

Deck, C. and Willinger, R. (2008) 'Improved head injury criteria based on head FE model', Int. J. Crashworthiness, Vol. 13, No. 6, pp.667-679.

Ewing, C., Thomas, D., Lustick, L., Muzzy III, W., Willems, G. and Majewski, P. (1977) 'Dynamic response of the human head and neck to +Gy impact acceleration', Proceedings of 21st Stapp Car Crash Conference, Society of Automotive Engineers, Warrendale, PA. Paper 770928 pp.549-586.

Ewing, C., Thomas, D., Patrick, L., Beeler, G. and Smith, M. (1968) 'Dynamic response of the head and neck of the living human to -Gx impact acceleration', Proceedings of 12th Stapp Car Crash Conference Society of Automotive Engineers, Warrendale, PA Paper 680792, pp.424-439.

International Traffic Safety Data and Analyse Group (IRTAD) (2004) Keeping Children Safe In Traffic - ISBN-92-64-10629-4 (C) OECD 2004. Available at: www.irtad.net.

Irwin, A. and Mertz, H.J. (1997) 'Biomechanical basis for the CRABI and hybrid III Child dummies', SAE Paper 973317.

Meyer, F., Bourdet, N., Deck, C., Willinger, R. and Raul, J.S. (2004) 'Human neck finite element model development and validation against original experimental data' Stapp Car Crash Conference. Nashville Paper: 2004-22-0008, Vol. 48, pp.177-206.

Meyer, F., Roth, S. and Willinger, R. (2008) 'Child neck FE model development and validation', Int. J. Human factors Modelling and Simulation, Vol. 1, No. 2, pp.244-257.

Nakahira, Y., Furukawa, K., Niimi, H., Ishihara, T., Miki, K. and Matsuoka F. (2000) 'A combined evaluation method and modified maximum likelihood method for injury risk curves', Proceedings of the IRCOBI Conference, pp.147-156.

Roth, S., Vappou, J., Raul, J.S. and Willinger, R. (2008) 'Child head injury criteria investigation through numerical simulation of real world trauma', Computer Methods and Programs in Biomedicine, Vol. 93, No. 1, pp.32-45.

Van Der Horst, M.J. (2002) 'Human head neck response in frontal, lateral and rear end impact loading - modeling and validation', $\mathrm{PhD}$ Thesis, Technical University of Eindhoven, Eindhoven, The Netherlands. 